\title{
Polimorfismo de hemoglobina em rebanho de ovinos Hampshire Down
}

\section{Hemoglobin polymorphism in Hampshire Down sheep herd}

\author{
Fabíola Cristine de Almeida Rego ${ }^{1 *}$; Flavio Antonio Barca Junior ${ }^{2}$; Luiz Fernando \\ Coelho Cunha Filho ${ }^{2}$; Werner Okano ${ }^{2}$; Luis César da Silva²; Agostinho Ludovico²; \\ Silvia Manduca Trapp ${ }^{2}$; Elsa Santana ${ }^{2}$; Neide Mariko Tanaka ${ }^{2}$
}

\begin{abstract}
Resumo
O presente trabalho teve como objetivo determinar os tipos de hemoglobinas em ovinos Hampshire Down e verificar se este locus encontra-se em equilíbrio de Hardy-Weinberg. Foram coletadas 46 amostras de sangue de ovinos Hampshire Down, considerados clinicamente sadios. Estas amostras foram utilizadas para a separação das hemoglobinas por eletroforese. A eletroforese das hemoglobinas revelou uma banda lenta caracterizada como hemoglobina $\mathrm{B}(\mathrm{HbBB})$, uma banda rápida caracterizada como hemoglobina $\mathrm{A}$ ( $\mathrm{HbAA}$ ) e duas bandas no heterozigoto para hemoglobinas A e B ( $\mathrm{HbAB})$. A variante $\mathrm{HbAB}$ foi a mais freqüentemente encontrada, seguida pela hemoglobina $\mathrm{A}(\mathrm{HbAA})$ e $\mathrm{B}(\mathrm{HbBB})$. A freqüência genotípica dos indivíduos $\mathrm{BB}, \mathrm{AB}$ e $\mathrm{AA}$ foram 36,$95 ; 54,35$ e 8,70\% respectivamente. A freqüência alélica de $\mathrm{A}$ e $\mathrm{B}$ foram respectivamente $35,87 \%$ e $64,13 \%$. Pelo teste do qui-quadrado realizado $\left(\chi^{2}=0,859\right.$ e $\left.p=0,6509\right)$ confirmou-se que o locus testado está em equilíbrio de Hardy-Weinberg.
\end{abstract}

Palavras-chave: Eletroforese, polimorfismo de hemoglobina, ovinos

\begin{abstract}
The present study aimed to determine the types of hemoglobin in Hampshire Down crossbreed sheep, and verify that this locus is in Hardy-Weinberg equilibrium. There have been collected 46 blood samples of healthy Hampshire Down crossbreed sheep. These samples were used to separate the hemoglobin per electrophoresis. The electrophoresis of the hemoglobin revealed a slow band characterized as hemoglobin $\mathrm{B}(\mathrm{HbBB})$, a fast band characterized as hemoglobin $\mathrm{A}(\mathrm{HbAA})$ and two bands in the heterozygous hemoglobin $\mathrm{A}$ and $\mathrm{B}(\mathrm{HbAB})$. The HbAB type was the most frequently one, followed by hemoglobin $\mathrm{A}(\mathrm{HbAA})$ and $\mathrm{B}(\mathrm{HbBB})$. The genotypic frequency of individuals $\mathrm{BB}, \mathrm{AB}$ and $\mathrm{AA}$ were 36,$95 ; 54,35$ and $8,70 \%$ respectively. The allelic frequency of $\mathrm{A}$ and $\mathrm{B}$ were respectively $35,87 \%$ and $64,13 \%$. The qui square test $\left(\chi^{2}=0.859\right.$ and $\left.p=0.6509\right)$ confirmed that the tested locus is in HardyWeinberg equilibrium.
\end{abstract}

Key words: Electrophoresis, hemoglobin polymorphism, sheep

1 Prof ${ }^{\mathrm{a}} \mathrm{Dr}^{\mathrm{a}}$ da Universidade Norte do Paraná, UNOPAR. Rua Eurilemos, n 1455, apto 205 A, CEP: 86708250, Arapongas, PR. E-mail: fabíola_rego@yahoo.com.br

2 Colaboradores, Docentes Universidade Norte do Paraná, UNOPAR. Arapongas, PR. E-mail: flavio.barca@unopar.br; luiz. cunha@unopar.br; vetwerner@gmail.com; silvaluizcesar@gmail.com; agostinho@rocketmail.com; smtrapp@gmail.com; elsa. santana@unopar.b; nmtanaka@onda.com.br

* Autor para correspondência 


\section{Introdução}

O estudo do polimorfismo da hemoglobina possui significância para a determinação de variantes presentes na população avaliada, bem como para o diagnóstico de variantes patológicas (NAOUM, 1997; ALVES et al., 2003). Além disto, a determinação da variante de hemoglobina pode ser um dos parâmetros genéticos empregados em estudos de identificação de indivíduos e de parentesco (SMITH, 1996).

Existem outros fatores que reforçama importância deste estudo, como por exemplo o fato de que o tipo de hemoglobina em ovinos pode estar relacionado com a resistência ao parasitismo por nematódeos gastrintestinais (BUVANENDRAN et al., 1981), bem como à respostas de desempenho animal, como a produção de carne ou produção de lã (DALLY et al., 1980). Além disso, sabe-se que algumas raças de ovinos apresentam resistência genética às infecções por nematódeos gastrintestinais (AMARANTE; AMARANTE, 2003).

Espera-se que as freqüências alélicas e genotípicas permanecem constantes dentro de uma população, desde que os acasalamentos ocorram ao acaso. A alteração deste equilíbrio irá ocorrer caso haja introdução ou retirada de indivíduos, mutação ou seleção dos animais para reprodução, sendo que este último iria oportunizar a certos componentes da população deixar maior número de descendentes (FALCONER, 1987). Fato este que para a produção animal é significativo, considerando a possibilidade de determinar genótipos favoráveis a certas características produtivas, seria possível então a escolha de animais com tais genótipos para serem os futuros reprodutores e genitores das gerações seguintes e desta forma propiciar maior produtividade pelo aumento da freqüência daquele alelo na população.

A diversidade dos tipos de hemoglobinas, nas diferentes raças de ovinos, é conhecida desde a década de 60 do século passado (EVANS et al., 1956; EVANS et al., 1958; ASHTON et al., 1967;
PIERAGOSTINI; ALLOGGIO; PETAZZI, 2010), os genótipos mais comumente identificados são HbAA, HbBB e HbAB (LACERDA; SOTO BLANCO, 2006; SOTOMAIOR; SOCCOL, 1998) e com menor freqüência encontra-se o alelo $\mathrm{HbC}$, normalmente relacionado em ovinos com quadro de anemia e proporcionando vantagem nos casos da existência de hipóxia tecidual, sendo nestes casos a hemoglobina A substituída pela C (JAIN, 1993; ESHARATAKHAH et al., 2007). Existe ainda a hemoglobina fetal, presente em animais recém nascidos, que permanece por um período curto na corrente sangüínea e é caracterizada por apresentar maior resistência osmótica que as demais (JAIN, 1993).

O presente trabalho teve por objetivo verificar a existência do polimorfismo de hemoglobina em um rebanho ovino da raça Hampshire Down.

\section{Material e Métodos}

Foi utilizado um rebanho de 46 ovinos da raça Hampshire Down, composto por matrizes com idade entre 1,5 a 3 anos, e borregas até um ano de idade. Este rebanho, considerado clinicamente sadio, é pertencente à região de Arapongas, Norte do Paraná.

Os testes foram realizados no Laboratório de Imunogenética Animal da Universidade Norte do Paraná (UNOPAR), Campus de Arapongas-PR.

As amostras de sangue foram colhidas por venopunção da veia jugular, utilizando-se sistema a vácuo, em tubos contendo anticoagulante citrato de sódio. Após a colheita, as amostras foram encaminhadas até o laboratório, onde foram mantidas refrigeradas em temperatura entre $2^{\circ} \mathrm{C}$ e $8^{\circ} \mathrm{C}$.

A amostra total de sangue foi "lavada" em solução fisiológica $0,89 \%$ por, no mínimo, três vezes, e após centrifugação obteve-se a papa de hemácias, que foi utilizada no preparo da solução de hemoglobina a $2,5 \%$. 
A determinação do tipo de hemoglobina foi realizada através de eletroforese (Figura 1) vertical em gel de poliacrilamida a $10 \%$, em $\mathrm{pH}$ alcalino $(\mathrm{pH} 8,3)$, com volume de $7 \mu \mathrm{L}$ para cada amostra, segundo técnica modificada de
Naoum (1999). Para a confecção do gel, utilizouse o mix de acrilamida 30\%, tampão Tris 3,77 M ( $\mathrm{pH} 8,9$ ), persulfato de amônio $20 \%$ e TEMED (Tetramethylethylenediamine).

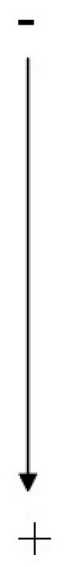

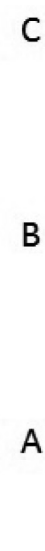

A

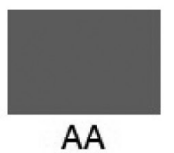

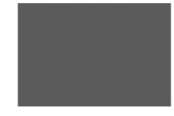
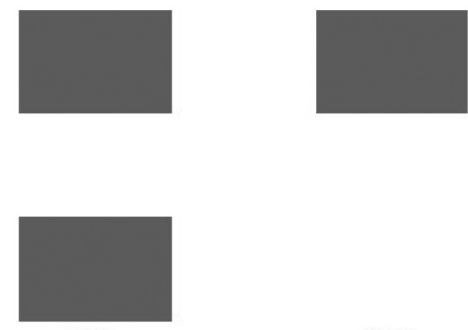

$\mathrm{AB}$

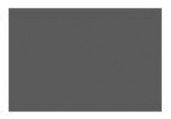

BB
$\mathrm{CC}$

Figura 1. Representação gráfica das bandas eletroforéticas e os tipos de hemoglobinas em ovinos.

Após a determinação do tipo de hemoglobina de cada animal, foi feito levantamento do número de indivíduos de cada genótipo e dessa forma foi possível calcular as freqüências genotípicas e alélicas para o locus da hemoglobina e verificar se o mesmo está em equilíbrio de Hardy-Weinberg. Seguindo os critérios recomendados por Falconer (1987) e sabendo que " $p+q=1$ " e " $p^{2}+2 p q+q^{2}=$ 1 " utilizou-se o teste de qui-quadrado para verificar se a população está em equilíbrio.

\section{Resultados e Discussão}

O teste de eletroforese das hemoglobinas apresentou uma banda lenta caracterizada como hemoglobina $\mathrm{B}(\mathrm{HbBB})$, uma banda rápida caracterizada como hemoglobina A (HbAA) e a presença das duas bandas nos animais heterozigotos ( $\mathrm{HbAB})$, seguiu-se a nomenclatura aceita internacionalmente pela International Society for Animal Geneticas (ISAG) (EVANS et al., 1956; EVANS et al., 1958; ASHTON et al., 1967).

No rebanho estudado a variante $\operatorname{HbAB}(54,35 \%)$ foi a mais encontrada, seguida pela $\operatorname{HbBB}(36,95 \%)$ e por fim a $\mathrm{HbAA}(8,7 \%)$, conforme (Tabela 1). Não foi evidenciada a presença da hemoglobina fetal, fato este justificado pela idade dos animais que compuseram a amostra serem superior a 12 semanas. Também não foi observada a presença de $\mathrm{HbC}$.

Tabela 1. Frequência genotípica absoluta e relativa do locus da hemoglobina em população de ovinos.

\begin{tabular}{ccc}
\hline Genótipo & Freqüência absoluta & Freqüência Relativa (\%) \\
\hline BB & 17 & 36,95 \\
AB & 25 & 54,35 \\
AA & 04 & 8,70 \\
\hline Total & 46 & 100,00 \\
\hline
\end{tabular}

Fonte: Rego et al. (2011). 
As mesmas variantes de hemoglobina também foram encontradas em diferentes raças de ovinos (LACERDA; SOTO BLANCO, 2006; SOTOMAIOR; SOCCOL, 1998, AYRES et al., 2009). Entretanto, em trabalho realizado por Esharatakhah et al. (2007) com raças ovinas iranianas, não observaram a presença da $\mathrm{HbBB}$, corroborando com as observações de Mohri, Jannatabadi e Aslani (2005). Já em outra pesquisa, também com raças iranianas, não verificou-se a ocorrência da HbAA (MOSTAGHNI, 1978). Esses resultados indicam que as freqüências dos tipos de hemoglobina estão relacionadas aos grupos raciais, já que este polimorfismo depende diretamente das freqüências dos alelos na população.

Esses resultados diferem aos encontrados por Lacerda e Soto Blanco (2006), apenas quanto aos animais homozigotos, que ao estudar uma população de 49 ovinos mestiços Santa Inês observou superioridade da variante $\mathrm{HbAB}$, que encontrava-se na proporção de $51 \%$, seguido pela $\operatorname{HbBB}(28,6 \%)$ e HbAA com 20,4\% da população. Trabalhando com um total de 296 animais de diversas raças nativas (Santa Inês, Rabo Largo e Morada Nova), uma de origem da África do Sul (Dorper) e seus cruzamentos, Ayres et al. (2009) encontraram maior número de animais com genótipo HbAA (49\%), seguidos por $\mathrm{HbAB}(39,18 \%)$ e finalmente $\mathrm{HbBB}$ $(11,82 \%)$, em nenhuma das raças identificou-se o alelo $\mathrm{HbC}$.

Em trabalho realizado no município de Santa Maria (RS), com ovinos Corriedale, foram encontrados 4,7\% do tipo HbAA; 29,7\% de animais com o genótipo $\mathrm{HbAB}$; e a maior percentagem $(65,6 \%)$ para o tipo HbBB (FAN; MOREIRA; FISCHE, 1981). Contudo valores diferentes dos obtidos de Weimer, Franco e Moraes (1984) que avaliaram também animais da raça Corriedale no mesmo estado e obtiveram freqüências 17,65\% (HbAA), 53,43\% (HbAB) e 28,92\% (HbB). Neste mesmo trabalho Weimer, Franco e Moraes (1984) também testaram animais da raça Romney Marsh, raça de origem inglesa assim como a Hampshire
Down, sendo as freqüências 18,01\% (HbAA), $49,69 \%$ ( $\mathrm{HbAB})$ e 32,30\% ( $\mathrm{HbBB})$, freqüências estas que assemelham-se a do presente trabalho apesar das raças serem diversas.

Utilizando as freqüências genotípicas verificadas no presente trabalho, foi possível determinar as freqüências alélicas, sendo $64,13 \%$ do alelo $\mathrm{HbB}$ e $35,87 \%$ do alelo $\mathrm{HbA}$, valores demonstrados na (Tabela 2).

Tabela 2. Frequência alélica para o locus da hemoglobina ovina.

\begin{tabular}{cc}
\hline Alelo & Freqüência Alélica (\%) \\
\hline A & 35,87 \\
B & 64,13 \\
\hline Total & 100,00 \\
\hline
\end{tabular}

Fonte: Rego et al. (2011).

Pelo teste do qui-quadrado realizado $\left(\chi^{2}=0,859 \mathrm{e}\right.$ $\mathrm{p}=0,6509$ ) confirmou-se que o locus testado está em equilíbrio de Hardy-Weinberg, indicando equilíbrio nas proporções genotípicas permitindo assim a comparação destas freqüências com as existentes em outras populações também em equilíbrio.

As freqüências alélicas do presente estudo foram diferentes dos resultados apresentados por Sotomaior e Socol (1998), que relataram a freqüência do alelo $\mathrm{HbA}$ de $6 \%$ e a do alelo $\mathrm{HbB}$ 94\%, em rebanho de ovelhas Suffolk na região de Curitiba, PR, raça de origem inglesa assim como a dos animais avaliados, tais valores estão muito próximos aos encontrados por Templetom, Price e Bogart (1972) que trabalharam com 95 animais também da raça Suffolk, sendo as freqüências $94,7 \%$ para o alelo B e 5,3\% para o alelo A.

Salako, Ijadunola e Agbesola (2007) estudando o polimorfismo de hemoglobina em rebanho ovino $(n=50)$ e caprino $(n=150)$, na região da Nigéria, verificaram a superioridade do alelo $\mathrm{HbB}$ em relação ao $\mathrm{HbA}$ em ambas as populações. Verificaram ainda, no rebanho caprino, a presença de um indivíduo 
com genótipo HbAC. Esta baixa freqüência do alelo $\mathrm{C}$, segundo o mesmo autor, pode estar relacionada com a maior incidência de anemia nos animais com tal genótipo, verificado também por Moore et al. (1966), que observaram que a presença do alelo $\mathrm{HbC}$ nos animais anêmicos era reflexo da substituição do alelo $\mathrm{HbA}$ pelo $\mathrm{HbC}$.

As diferenças encontradas nas freqüências dos alelos entre o presente trabalho e os demais podem ser creditadas por avaliarem diferentes raças, algumas com diferentes origens genéticas e geográficas. São ainda influenciadas pelos objetivos da criação (programa de melhoramento) e a pressão de seleção exercida sobre os rebanhos. A ausência do alelo $\mathrm{HbC}$ pode ser justificada por todos animais testados estarem em perfeitas condições clínicas e sanitárias, desta forma sem o possível fator predisponente da anemia para a ocorrência da substituição do alelo $\mathrm{HbA}$ pelo $\mathrm{HbC}$.

Conclui-se que o locus de hemoglobina para os ovinos da raça Hampshire Down testados, apresentou polimorfismo, com a presença dos alelos $\mathrm{HbA}$ e $\mathrm{HbB}$ e as três combinações possíveis de genótipos, sendo o mais freqüente a $\mathrm{HbAB}$, seguido por $\mathrm{HbBB}$ e HbAA. Possíveis associações de características produtivas a este locus poderiam ser temas de futuras pesquisas, o que permitiria, desde que confirmada a associação, a seleção de animais com determinado genótipo visando o aumento da produtividade.

\section{Referências}

ALVES, R. T.; MATTOS, L.C .; FERRARI, F.; BONINIDOMINGUES, C. R. Avaliação do polimorfismo de grupos sangüíneos e fenótipo de hemoglobinas em um grupo de universitários de São José do Rio Preto, SP. Revista Brasileira de Hematologia e Hemoterapia, São José do Rio Preto, v. 25, n. 1, p. 65-71, 2003.

AMARANTE, A. F. T.; AMARANTE, M. R. V. Breeding sheep for resistance to nematode infections. Journal of Animal and Veterinary Advances, Medwell, v. 2, n. 3, p. $147-161,2003$.
ASHTON, G. C.; GILMOUR, D. G.; KIDDY, C. A.; KRISTJANSSON, F. K. Proposals on nomenclature of protein polymorphisms in farm livestock, Genetics, Pittsburgh, v. 56, n. 3, p. 353-362, 1967.

AYRES, M. C. C.; CHAVES FILHO, R. M.; ROCHA, J. S.; GAMA, S. M. S.; CAVALCANTI, A. S. R.; ALMEIDA, M. Â. O. ; BITTENCOURTT, T. C. B. S. C. Polimorfismo da hemoglobina de ovinos (Ovis aries, L.): determinação do tipo e da freqüência em animais criados no Estado da Bahia. Revista Brasileira de Saúde e Produção Animal, Salvador, v. 10, n. 3, p. 637-644, 2009.

BUVANENDRAN, V.; SOORIYAMOORTHY, T.; OGUNSUSI, R. A.; ADU, I. F. Haemoglobin polymorphism and resistance to helminths in red Sokoto goats. Tropical Animal Health and Production, Boston, v. 13, p. 217-221, 1981.

DALLY, M. R.; HOHENBOKEN, W.; THOMAS, D. L.; CRAIG, A. M. Relationships between hemoglobin type and reproduction, lamb, wool and milk production and health-related traits in crossbred ewes. Journal of Animal Science, Champaign, v. 50, n. 3, p. 418-427, 1980.

ESHARATAKHAH, B.; SAFI, S.; ZHAKI, P.; NIA, P. K.; SADAGHIAN, M. Study in haemoglobin polymorphism in two breeds of Iranian sheep. Journal of Animal and Veterinary Advances, Medwell, v. 6, n. 12, p. 1426-1428, 2007.

EVANS, J. V.; HARRIS, H.; WARREN, F. L. The distribution of hemoglobin and blood potassium types in British breeds of sheep. Proceedings of the Royal Society of London. Series B, Biological Sciences, London, v. 149, n. 935 , p. $249-262$, 1958 .-

EVANS, J. V.; KING, J. W. B.; COHEN, B. L.; HARRIS, H.; WARREN, F. L. Genetics of haemoglobin and blood potassium differences in sheep. Nature, London, v. 178, n. 4538, p. 849-850, 1956.

FALCONER, D S. Introdução a genética quantitativa. Viçosa: UFV, 1987. 279 p.

FAN, L. C. R.; MOREIRA, E. C.; FISCHER, R. Freqüência dos tipos de hemoglobina em ovinos adultos no município de Santa Maria. Revista Centro de Ciências Rurais, Santa Maria, v. 11, n. 1, p. 7-11, 1981.

JAIN, N. C. Essentials of veterinary hematology. Philadelphia: Lea \& Fabinger, 1993. 417 p.

LACERDA, R. M.; SOTO-BLANCO, B. Determinação das variantes de hemoglobina em ovinos mestiços Santa Inês. Caatinga, Mossoró, v. 19, p. 345-349, 2006. 
MOHRI, M.; JANNATABADI, A. A.; ASLANI, M. R. Studies on haemoglobin polymorphism of two breeds of Iranian sheep and its relationship to concentration of iron, copper, haemoglobin and $\mathrm{RBC}$ number. Veterinary Research Communications, Amsterdam, v. 29, n. 4, p. 305-312, 2005.

MOORE, S. L.; GODLEY, W. C.; VAN VLIET, G.; LEWIS, J. P.; BOYD, E.; HUISMAN, T. H. J. The Production of hemoglobin $\mathrm{c}$ in sheep carrying the gene for hemoglobin A: Hematologic Aspects. Blood, Washington, v. 28, n. 3, p. 314-329, 1966.

MOSTAGHNI, K. Studies on haemoglobin variants and some blood minerals of different breeds of Iranian sheep. Journal of Veterinary Medicine, Berlin, v. 25, n. 8, p. 647-651, 1978.

NAOUM, P. C. Hemoglobinopatias e talassemias. São Paulo: Sarvier, 1997. 171 p.

. Eletroforese. Técnicas e diagnósticos. 2. ed. São Paulo: Livraria Santos Editora. 1999. 154 p.

PIERAGOSTINI, E.; ALLOGGIO, I.; PETAZZI, F. Insights into hemoglobin polymorphism and related functional effects on hematological pattern in mediterranean cattle, goat and sheep. Diversity, Switzerland. v. 2, n. 4, p. 679-700, 2010.
SALAKO, A. E.; IJADUNOLA, T. O.; AGBESOLA, Y. O. Hemoglobin polymorphism in Nigerian indigenous small ruminant populations - preliminary investigation. African Journal of Biotechnology, v. 6, n. 22, p. 2636-2638, 2007. Disponível em: <http://www.academicjournals. org/AJB ISSN 1684-5315 (C) 2007 Academic Journals> . Acesso em: 20 abr. 2009.

SMITH, B. P. Tratado de medicina interna de grandes animais. São Paulo: Manole, 1996. 1738 p.

SOTOMAIOR, C. S.; SOCCOL, V. T. Estudo do tipo de hemoglobina como auxiliar na seleção de ovinos resistentes e susceptíveis aos helmintos gastrintestinais. Archives of Veterinary Science, Curitiba, v. 3, n. 1, p. 5155, 1998.

TEMPleTOM, J. W.; PRICE, D.; BOGART, R. Frequency of hemoglobin types in five breed of sheep. Journal Heredity, Oxford, v. 63, n. 4, p. 202-204, 1972.

WEIMER, T. A.; FRANCO, M. H. P.; MORAES, J. C. F. Hemoglobin and transferrin types in Corriedale and Romney Marsh sheep in Brazil. Brazilian Journal of Genetics, Ribeirão Preto, v. 7, n. 2, p. 287-297, 1984. 